\title{
DIVERSIDAD Y COMPLEJIDAD EN EL FENÓMENO DE LAS DROGAS: EL POLICONSUMO SIMULTÁNEO EN ESTUDIANTES UNIVERSITARIOS EN UNA UNIVERSIDAD, CUNDINAMARCA - COLOMBIA
}

\author{
Rosibel Prieto Silva ${ }^{1}$, Laura Simich ${ }^{2}$, Carol Strike ${ }^{3}$,Bruna Brands ${ }^{4}$, Norman Giesbrecht ${ }^{5}$, Akwatu Khenti ${ }^{6}$
}

\footnotetext{
${ }^{1}$ MSc. Ph.D. Profesora Asociada. Departamento Salud de Colectivos. Facultad de Enfermería. Universidad Nacional de Colombia. Colombia. E-mail: rprietos@unal.edu.co

${ }^{2}$ Ph.D. Researcher. Health Systems Research and Consulting Unit. Centre for Addiction and Mental Health. University of Toronto. Toronto, Canada. E-mail: laura_simch@camh.net

${ }^{3}$ Ph.D. Researcher. Health Systems Research and Consulting Unit. Centre for Addiction and Mental Health. University of Toronto. Toronto, Canada. E-mail: carol_strike@camh.net

${ }^{4}$ Ph.D. Researcher. Office of Research and Surveillance. Drug Strategy and Controlled Substances Programme. Health Canada and Public Health and Regulatory Policies. Center for Addiction and Mental Health. University of Toronto. Toronto, Canada. E-mail: bruna_brands@camh.net

${ }^{5}$ Ph.D. Researcher. Health Systems Research and Consulting Unit. Centre for Addiction and Mental Health. University of Toronto, Canada. E-mail: norman_giebsbrecht@camh.net

${ }^{6}$ MSc. Director of International Office. Centre for Addiction and Mental Health. Toronto, Canadá. E-mail: akwatu_khenti@ camh.net
}

RESUMEN: El policonsumo simultáneo de sustancias psicoactivas es un tópico que diversifica y complejiza el fenómeno de las drogas en nuestra sociedad contemporánea. Se realizó un estudio cuantitativo transversal, con el propósito de describir los patrones de policonsumo simultáneo de sustancias psicoactivas, con una muestra de 975 estudiantes de pregrado de las carreras relacionadas con el área de la salud, en una Universidad en Cundinamarca, Colombia. Se observó un alto reporte de estudiantes que realizan policonsumo simultáneo, destacándose la combinación de alcohol + tabaco. Se evidenciaron comportamientos diversos del policonsumo, relacionados con la edad de inicio, el reporte del momento del consumo, el año académico cursado, las sustancias empleadas y la transición del consumo al policonsumo simultáneo. Se sugiere incluir este tópico en la formación de recursos humanos en salud y en el desarrollo de investigación.

DESCRIPTORES: Estudiantes. Drogas ilícitas. Conducta adictiva. Transtornos relacionados con sustancias. Factores de riesgo.

\section{DIVERSITY AND COMPLEXITY IN THE PHENOMENON OF DRUGS: SIMULTANEOUS POLYDRUG USE IN UNIVERSITY STUDENTS IN ONE UNIVERSITY, CUNDINAMARCA - COLOMBIA}

\begin{abstract}
The simultaneous polydrug use is a topic that diversifies and increase complexity in the drug phenomenon in our contemporary society. We conducted a quantitative cross-sectional study, in order to describe patterns of simultaneous polydrug use of psychoactive substances, within a sample of 975 undergraduate students in health sciences, at one university in Cundinamarca, Colombia. We observed a high rate of report in simultaneous poly drug use, especially the combination of alcohol-tobacco. We identified different behaviors related to starting age, occasion for consumption, academic year, substances used, and the transition from single to poly drug use. We recommended the inclusion of this topic in training of health care human resources and in further research.

DESCRIPTORS: Students. Street drugs. Behavior addictive. Substance-Related disorders. Risk factors.
\end{abstract}

\section{DIVERSIDADE E COMPLEXIDADE NO FENÔMENO DAS DROGAS: O POLICONSUMO SIMULTÂNEO EM ESTUDANTES UNIVERSITÁRIOS, CUNDINAMARCA - COLOMBIA}

\begin{abstract}
RESUMO: A utilização simultânea de mais de uma substância psicoativa, ao mesmo tempo é um tema que diversifica e complexifica o fenômeno das drogas na sociedade contemporânea. Foi realizado um estudo quantitativo e transversal, a fim de descrever os padrões de policonsumo simultâneo de substâncias psicoativas entre 975 estudantes de graduação da área da saúde, em uma universidade em Cundinamarca, Colômbia. Foi observado um alto índice de estudantes da amostra que relataram o uso simultâneo de múltiplas drogas, destacando a combinação de álcool e tabaco. Diferentes comportamentos são evidentes na utilização simultânea de múltiplas drogas, relacionadas com a idade de início do uso; do consumo de múltiplas drogas; o ano letivo estudado; as substâncias utilizadas no consumo de múltiplas drogas e à transição do consumo para o policonsumo de drogas. Sugerem a inclusão deste tema na formação de recursos humanos em saúde e na pesquisa.
\end{abstract}

DESCRITORES: Estudantes. Drogas ilícitas. Comportamento aditivo. Transtornos relacionados ao uso de substâncias. Fatores de risco. 


\section{INTRODUCCIÓN}

El fenómeno de las drogas ha sido estudiado ampliamente a nivel mundial, lo que ha demostrado su comportamiento complejo y la diversidad de abordajes a esta problemática. A nivel latinoamericano el estudio de este fenómeno ha tenido un gran liderazgo por parte de la CICAD con el Programa Internacional en Investigación para Profesionales de la Salud y Áreas Relacionadas para estudiar el fenómeno de las Drogas en América Latina. ${ }^{1}$ El Informe Mundial sobre las Drogas 2008, elaborado por la Oficina de las Naciones Unidas contra la Droga y el Delito (ONUDD), informa acerca del incremento del consumo de sustancias psicoactivas en el mundo y especialmente en América Latina. Se estima que 208 millones de personas usan sustancias psicoactivas, lo que equivale a un $4.9 \%$ de la población mundial; el rango de edad entre los consumidores se encuentra entre los 15 y 64 años. ${ }^{2}$

Uno de los abordajes de este fenómeno, ha sido el estudio en relación a las sustancias de consumo, entre los que se destaca un mayor número de estudios sobre drogas legales y entre estas con mayor énfasis en relación a las sustancias alcohol y tabaco, y su participación en la causalidad de enfermedades cardiacas, pulmonares y diversidad de neoplasias.

El fenómeno de las drogas últimamente se ha complejizado aún más, se observa un aumento del consumo de las drogas y una menor edad para el inicio del consumo, a pesar de los esfuerzos de los gobiernos y de las entidades encargadas. De igual manera, el consumo de sustancias se ha diversificado, y se observa no solo el consumo de más de una sustancia psicoactiva, lo que se ha denominado policonsumo, sino que se observa el consumo de más de una sustancia psicoactiva al mismo tiempo, lo que se ha denominado policonsumo simultáneo. ${ }^{3}$ Este patrón de policonsumo simultaneo destaca entre otras su importancia de estudio por varios factores, entre ellos el incremento en las dosis, la toxicidad y las interacciones entre las sustancias psicoactivas usadas.

En el estudio, "Desaprovechar lo mejor y lo de mayor capacidad: el abuso de sustancias en las universidades americanas", ${ }^{4}$ refleja el alcohol como la principal droga usada en las universidades (65\%-70\%). El fenómeno del abuso de sustancias psicoactivas va mas allá del alcohol incluyendo otras drogas lícitas e ilícitas. La tendencia identificada en las últimas décadas se ha intensificado y los estudiantes están inmersos en una cultura de abuso de sustancias adictivas con consecuencias en los ámbitos de la salud, académico y social, involucrando a toda la comunidad universitaria. A pesar de que la droga de mayor consumo en las universidades es el alcohol $49.4 \%$, el uso de otras sustancias lícitas e ilícitas ha aumentado. En la actualidad, los estudiantes que usan marihuana y otras drogas ilícitas se ha incrementado con porcentajes desde $1.9 \%$ a $4.0 \%$, mientras que el uso de otras sustancias como la cocaína ha aumentado en un $52 \% .^{4}$

Otros autores confirman la importancia de estudiar el policonsumo de sustancias psicoactivas en estudiantes universitarios ${ }^{5-6}$ y en especial en el área de la salud, ${ }^{7-8}$ por ser considerada una población vulnerable. La transición del colegio a la universidad puede ser una experiencia muy estresante. La nueva experiencia para algunos estudiantes de vivir en forma independiente y con menos control paterno, los hace susceptibles a un mayor riesgo de abuso/uso de sustancias. ${ }^{9}$ La vida universitaria es con frecuencia una cultura en sí misma, con muchas oportunidades para experimentar el consumo de varias sustancias a las cuales no se había estado expuesto anteriormente, como es en el caso de "fiestas" desenfrenadas dentro o fuera de la universidad, y la promoción que se hace del uso del alcohol y otras sustancias para disminuir el estrés y divertirse. ${ }^{9}$

Se encuentran algunos estudios sobre policonsumo y algunos pocos sobre policonsumo simultaneo de sustancias psicoactivas, no obstante estos han sido realizados en otros contextos geográficos y socioculturales. Todo lo cual confirma la necesidad e importancia de conocer la problemática del policonsumo simultaneo de sustancias psicoactivas en el contexto latinoamericano, y en particular en el contexto universitario colombiano.

Este artículo tiene por objeto describir los patrones de policonsumo simultáneo de sustancias psicoactivas en estudiantes de pregrado de el área de la salud de una universidad de Bogotá, Colombia, específicamente en relación a la edad, año universitario actualmente cursado y sustancias de policonsumo.

\section{MÉTODO}

Los datos presentados aquí se refieren a una investigación cuantitativa, de corte trans- 
versal. Este estudio fue parte de un proyecto multicéntrico de exploración del fenómeno de policonsumo simultáneo de sustancias psicoactivas en estudiantes universitarios y en donde participaron seis países de América Latina y un país del Caribe. Este artículo se refiere a los hallazgos del estudio en una universidad en Cundinamarca, Colombia.

La muestra de interés estuvo compuesta por un total de 975 estudiantes de pregrado, quienes se encontraban cursando el primer y segundo año de carreras relacionadas con el área de la salud al momento de realización del estudio. La recolección de los datos se llevo a cabo en el primer semestre del año 2009. La participación fue anónima y voluntaria, previa entrega escrita de información del estudio y del diligenciamiento de consentimiento informado. Con el fin de que todos los estudiantes elegibles tuvieran igual oportunidad de participar, se seleccionaron aleatoriamente cursos obligatorios de cada carrera y en cada año de estudio. Para la recolección de los datos se diseñó un cuestionario auto-administrado, con 58 preguntas; el cual se sometió a pruebas de validez y confiabilidad. La recolección de los datos se realizó en los salones de clases, en ausencia del profesor responsable del curso, y bajo la conducción del investigador principal. Con el fin de garantizar la participación de forma anónima, la confidencialidad y seguridad de los documentos, luego del diligenciamiento del consentimiento informado, el estudiante debió depositarlo en una urna cerrada y sellada, y en otra urna con las mismas características, depositó el instrumento diligenciado. El cuestionario permitió a los participantes proporcionar información adicional en caso necesario.

Los datos fueron sometidos a análisis de estadística descriptiva. Para el análisis de datos se utilizó el programa estadístico SPSS, versión 15.0. Este estudio fue aprobado por el Research Ethics Board (REB) del Centre for Addiction and Mental Health (CAMH) (Protocol reference \#225/2008) y por el Comité de Etica en Investigación de la universidad colombiana participante en el estudio.

\section{RESULTADOS}

\section{Características de la muestra y consumo de sustancias psicoactivas}

La muestra estuvo compuesta por un total de 975 estudiantes. La edad tuvo una mediana de 19 años, con un rango entre 16 y 40 años. Un $47.1 \%$ y $52.9 \%$ se encontraba cursando el primer y segundo año académico respectivamente. El $73.9 \%$ de los estudiantes en la muestra reportó haber usado alguna sustancia psicoactiva alguna vez en su vida y el $65.3 \%$ reporto haberlo hecho en los últimos 12 meses. Un 19.7\% reportó policonsumo simultáneo en los últimos 12 meses, cifra que alcanzo $12.4 \%$ en los últimos 30 días. Entre quienes reportaron algún tipo de consumo, el $26.6 \%$ reportó policonsumo simultáneo en los últimos 12 meses y el $16.8 \%$ en los últimos 30 días. Estas cifras alcanzan $30.1 \%$ y $19.0 \%$ entre los consumidores de cualquier sustancia en los últimos 30 días. La edad de inicio en el uso de sustancias psicoactivas fue de 14.5 años en promedio, con un rango entre 10 y 20 años. Este promedio de edad se elevó a 16.3 años en el caso de policonsumo simultáneo. Un $10.7 \%$ de los estudiantes de primer año refirió policonsumo, cifra que subió a $13.5 \%$ entre estudiantes de segundo año.

\section{Sustancias psicoactivas que conforman los patrones de policonsumo simultáneo}

En cuanto al patrón de policonsumo simultáneo, la combinación de cigarrillo y alcohol fue la más frecuente, con un $38 \%$ y $83 \%$ de los que reportaron policonsumo en los últimos 12 meses y 30 días respectivamente. Las combinaciones del policonsumo simultáneo en los últimos 12 meses fueron las siguientes (Figura 1): alcohol y marihuana, $13 \%$; alcohol, tabaco y marihuana, $8 \%$ ; tabaco y marihuana, $7 \%$; alcohol y cocaína, $5 \%$; alcohol, cocaína y tabaco, 3\%; alcohol, cocaína, marihuana y tabaco, $2 \%$; alcohol y medicamentos de prescripción, $2 \%$; marihuana y cocaína, $2 \%$; marihuana y medicamentos de prescripción, $1 \%$; tabaco y medicamentos de prescripción, $1 \%$. 


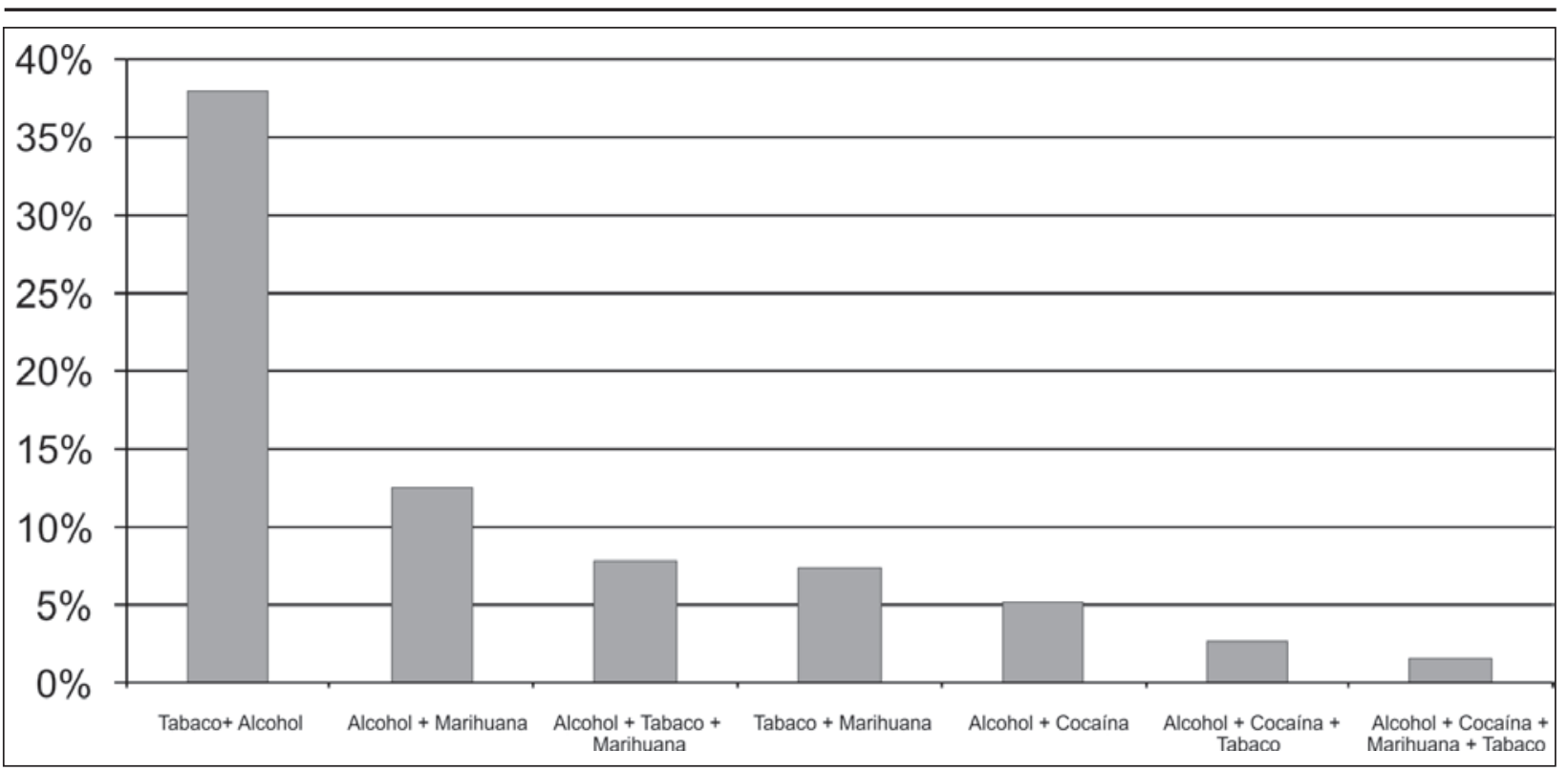

Figura 1- Patrones de sustancias psicoactivas utilizadas en el policonsumo simultáneo en los últimos 12 meses entre estudiantes universitarios. Cundinamarca Colombia, 2009

Las combinaciones del policonsumo simultáneo en los últimos 12 meses fueron las siguientes (Figura 2): alcohol y marihuana, 19\%; alcohol, tabaco y marihuana, $13 \%$; tabaco y marihuana, $12 \%$; alcohol y cocaína, $2 \%$; alcohol, cocaína y tabaco, $3 \%$; alcohol, cocaína, marihuana y tabaco, $2 \%$; alcohol y medicamentos con prescripción, $3 \%$; marihuana y cocaína, $2 \%$; marihuana y medicamentos con prescripción, 3\%; tabaco y medicamentos con prescripción, $2 \%$. Otras combinaciones planteadas que registraron respuestas positivas incluyeron: alcohol, cocaína, marihuana y tabaco; alcohol y medicamentos de prescripción; marihuana y medicamentos de prescripción; tabaco y medicamentos de prescripción; además de diversas combinaciones reportadas como respuestas abiertas.

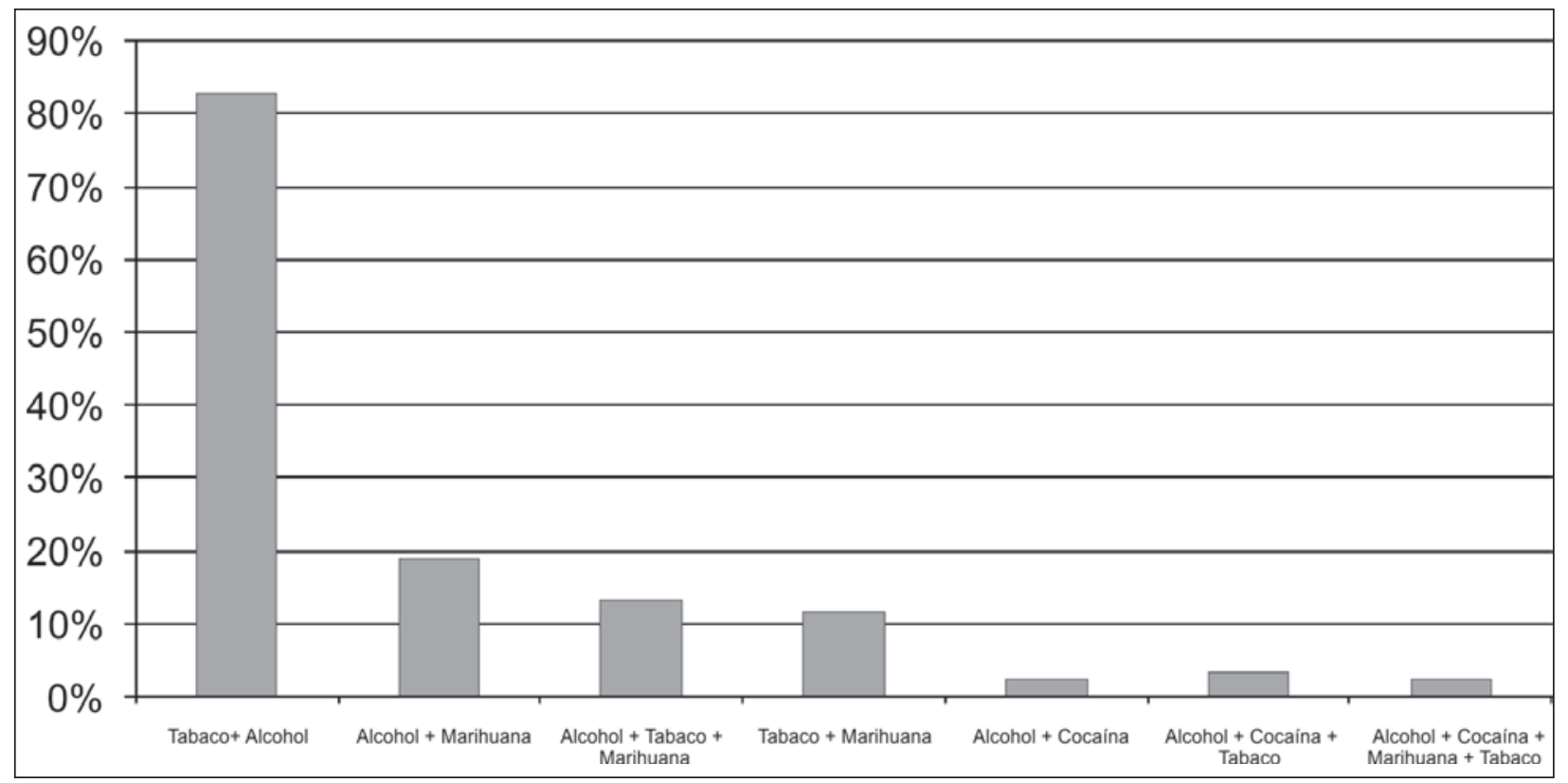

Figura 2 - Patrones de sustancias psicoactivas utilizadas en el policonsumo simultáneo en los últimos 30 días entre estudiantes universitarios. Cundinamarca Colombia, 2009 


\section{DISCUSIÓN}

El policonsumo simultáneo de sustancias psicoactivas es un tema en el cual no se dispone de suficiente información a nivel nacional. Los resultados presentados en este artículo permiten una aproximación al conocimiento de este tópico que se inserta en el estudio macro del fenómeno de las drogas.

Este estudio se suma a otros estudios que a nivel internacional se han desarrollado en este tópico. ${ }^{8,10-11}$ Actualmente en Colombia, no se dispone de información que referencie en específico la situación de policonsumo simultáneo de sustancias psicoactivas en estudiantes universitarios delárea de la salud. El Estudio Nacional de Consumo de Sustancias Psicoactivas en Colombia, $2008^{12}$ y la Encuesta Nacional sobre Consumo de Sustancias Psicoactivas en Jóvenes entre 10-24 años, ${ }^{12}$ reportan como sustancias psicoactivas de mayor consumo, el alcohol y el tabaco. En el estudio mencionado, el consumo de alcohol presenta las siguientes prevalencias: $86.08 \%$ en la vida, $61.18 \%$ en el último año y $34.77 \%$ en el último mes. El consumo de tabaco presenta las siguientes prevalencias: $44.49 \%$ en la vida, $21.46 \%$ en el último año y $17.06 \%$ en el último mes. Estas cifras son cercannas a las reportadas en el presente estudio. Adicionalmente, nosotros observamos una prevalencia importante de policonsumo simultáneo en estudiantes de pregrado en carreras de la salud en una universidad, tanto en los últimos 12 meses como 30 días.

La transición del consumo de una sustancia al policonsumo simultaneo podría incrementar la carga de toxicidad para el cuerpo humano, así como las implicaciones familiares, sociales y legales.

Las edades de inicio de consumo y policonsumo encontradas en nuestro estudio son en general concordantes con las de la literatura. ${ }^{13-16}$ Lo mismo ocurre con la combinación de alcohol y tabaco, que fue la más reportada por estudiantes en nuestro estudio. Varios estudios reportan el consumo y policonsumo de alcohol, tabaco y marihuana, como las de mayor prevalencia en estudiantes del área de la salud. ${ }^{7,17-18}$ Adicionalmente, en nuestro estudio destacan otras combinaciones utilizadas con frecuencia, principalmente las de alcohol y marihuana; alcohol, tabaco y marihuana; tabaco y marihuana; alcohol y cocaína; y alcohol, cocaína y tabaco. La literatura privilegia el estudio de la combinación entre alcohol y tabaco, y en menor medida de de alcohol y marihuana. Nuestro estudio contribuye por lo tanto a dar una visión más completa del problema de policonsumo simultáneo.
La literatura hace referencia al uso de sustancias psicoactivas como alcohol, tabaco y marihuana, solas o combinadas, como factor de riesgo para la transición hacia el consumo de otras sustancias, como la cocaína, ${ }^{10,19-21}$ lo cual ha sido definido en otros estudios como "gateway model". ${ }^{11}$

En este escenario de diversidad de sustancias psicoactivas y su policonsumo simultaneo, es necesario establecer las implicaciones para la salud humana, lo cual se sugiere profundizar a partir de los hallazgos de este estudio. Las consecuencias sociales e implicaciones legales del policonsumo simultaneo de sustancias psicoactivas, sin duda están estrechamente ligadas con los hallazgos de estudios de implicaciones legales, sociales y familiares, descritos en la literatura y relacionados con el consumo. ${ }^{22-24} \mathrm{El}$ consumo de sustancias psicoactivas puede asociarse a una variedad de consecuencias negativas, incluyendo un aumento en el riesgo de consumo de drogas años más tarde, fracaso escolare irresponsabilidad que pueden poner al adolescente en riesgo de accidentes, violencia, relaciones sexuales no planificadas e inseguras, así como suicidio. ${ }^{25}$

\section{CONCLUSIONES}

Con los hallazgos presentados en este artículo, se evidencian comportamientos diversos del policonsumo simultáneo de sustancias psicoactivas respecto al reporte del momento del consumo, a la edad de inicio del policonsumo, al año universitario cursado, a las sustancias empleadas en el policonsumo y la transición del consumo al policonsumo simultaneo de sustancias psicoactivas. Las cifras encontradas respaldan la relevancia del fenómeno de las drogas y del policonsumo simultáneo en estudiantes universitarios, por lo que consideramos que este debe ser un tópico prioritario en las agendas de la salud pública y de la salud individual de los adolescentes y jóvenes del país. Es tarea para los formadores de recursos humanos e investigadores del área de la salud el abordar este tópico, así como el aportar al fortalecimiento de la investigación para la generación de nuevos conocimientos en el área.

\section{Limitaciones}

Los resultados obtenidos en este estudio no son generalizables a la población de jóvenes en general o a la de estudiantes universitarios, ya que sólo se incluyeron estudiantes de primero y segundo años de carreras de ciencias de la salud en una universidad en Colombia. 


\section{Recomendaciones}

Consideraos importante la realización de estudios similares en otras instituciones de educación superior del país, para alcanzar conclusiones mas generalizables a la realidad universitaria del país. Así mismo, consideramos importante la extensión de estos estudios a estudiantes en edad escolar, que es donde el consumo y policonsumo de sustancias psicoactivas comienza con mayor frecuencia. Por último, considerando que esta problemática no sólo afecta a universitarios o a la población joven, se requiere profundizar el análisis de otros segmentos de la población, con el objeto de conocer y comprender mejor este fenómeno en el contexto colombiano.

\section{AGRADECIMIENTOS}

Al gobierno de Canadá/DFAIT, a la Organización de los Estados Americanos (OEA), Comisión Interamericana para el Control del Abuso de Drogas (CICAD), al Centre for Addiction and Mental Health, (CAMH-Toronto/Canadá). Así mismo se agradece a los estudiantes de pregrado que participaron en el estudio y a todas las personas que colaboraron de forma directa o indirecta en la implementación del estudio. Un agradecimiento especial al Dr. Gustavo Mery, CAMH Reviewer, por su colaboración en la revisión y editoración final de todos los trabajos y informes del grupo III.

\section{REFERENCIAS}

1. Wright MGM, Gliksman L, Khenti A, Furegato ARF. Investigación sobre el fenómeno de las drogas bajo el abordaje de los estudios multicéntricos en América Latina y Caribe. Rev Latino-am Enfermagem. 2009; 17(Esp):759-61.

2. Austria EUV. Se incrementa el uso de drogas en el mundo - ONU. El siglo de Torreon. 2008 Jun 27.

3. McCabe SE, Cranford JA, Morales M, Young A. Simultaneous and concurrent poly-drug use of alcohol and prescription drugs: prevalence, correlates, and consequences. J Stud Alcohol. 2006 Jul; 67(4):529-37.

4. Casa NC. Wasting the best and the brightest: substance abuse at America's Colleges and Universities. New York (US): Columbia University - National Center on Addiction and Substance Abuse; 2007.

5. Laranjo TS. University residence halls: socialization processes and drug consumption. Rev Saude Pública. 2006 Dez; 40(6):1027-34.

6. Simons J, Gaher R, Correia C, Hansen CC. Affectivemotivational model of marijuana and alcohol problems among college students. Psychol Addict Behav. 2005 Sep; 19(3):326-34.

7. Urrego D. Consumo de sustancias psicoactivas en estudiantes de especialidades médicas, Bogotá 2001. Rev Salud Pública. 2002 Jan; 4(1):59-73.

8. Oliveira EB, Furegato ARF. El trabajo del estudiante de enfermería como un factor de riesgo para el consumo de alcohol y otras drogas. Rev Latino-am Enfermagem. 2008 Jul-Ago; 16(Spe):565-71.

9. Pillon S, O'Brien B, Chavez K. The relationship between drugs use and risk behaviors in Brazilian university students. Rev Latino-am Enfermagem. 2005 Nov-Dez; 13(Spe):1169-76.

10. Barrett S, Darredeau C, Pihl R. Patterns of simultaneous poly-substance use in drug using university students. Hum Psychopharmacol. 2006 Jun; 21(4):255-63

11. Herrera-Vázquez M, Wagner FA, VelascoMondragón E, Borges G, Lazcano-Ponce E. Inicio en el consumo de alcohol y tabaco y transición a otras drogas en estudiantes de Morelos, México. Salud Públ Méx. 2004 Mar-Apr; 46(2):132-40.

12. Ministerio del Interior y de Justicia (CO). Dirección Nacional de Estupefacientes. Colombia 2009. [acceso 2009 Sept 20] Disponible en: http:/ / scholar.google. $\mathrm{com} /$ scholar?hl=es\&q=encuesta+nacional+de+200 $1+e n+j \%$ C3 \% B3venes+escolarizados+colombiano $\mathrm{s}+\mathrm{de}+10+\mathrm{a}+24+\mathrm{a} \% \mathrm{C} 3 \%$ B1os.\&btnG $=$ Buscar\&lr $=\&$ as_ylo $=\& a s \_v i s=0$

13. Martínez-Mantilla JA, Amaya-Naranjo W, Campillo HA, Rueda-Jaimes G, Campo-Arias A, DíazMartínez LA. Consumo de sustancias psicoactivas en adolescentes, Bucaramanga, Colombia, 19962004. Rev Salud Pública. 2007 Apr; 9(2):215-29.

14. Kosterman R, Hawkins JD, Guo J, Catalano RF, Abbott RD. The dinamics of alcohol and marihuana iniciation: patterns and predictors of first use in adolescence. Am J Public Health. 2000 Mar; 90(3):360-6.

15. Jordán Jinez ML, Souza JRM, Pillon SC. Drug use and risk factors among secondary students. Rev Latino-am Enfermagem. 2009 Mar-Apr; 17(2):246-52.

16. Pechansky F, Szobot CM, Scivoletto S. Alcohol use among adolescents: concepts, epidemiological characteristics and etiopatogenic factors. Rev Bras Psiquiatr. 2004; 26(Supl I):14-7.

17. López M, Santín C, Torrico E, Rodriguez J. Consumo de sustancias-psicoactivas en una muestra de jóvenes universitarios. Psicol Salud. 2003 Ene-Jun; 13(1):5-18.

18. Viña C, Herrero M. El consumo de sustancias psicoactivas en estudiantes de psicología de la Universidad de la Laguna. Interl J Clin Health Psichol. 2004; 4(3):521-36.

19. Liccardo R. Adolescent alcohol and marijuana comsumption: is there really a gateway effect? 1998 [acceso 2009 Sept 20]. Available from http:/ / www. nber.org/papers/w6348 
20. Smucker Barnwell S, Earleywine M. Simultaneous alcohol and cannabis expectancies predict. Subst Abuse Treat Prev Policy. 2006 Oct 11 [acceso 2009 Sept 20]; 1:29. Available from http://www. substanceabusepolicy.com/content/1/1/29

21. Duncan SC, Duncan TE, Hops H. Progressions of alcohol, cigarette, and marijuana use in adolescence. J Behav Med. 1998 Aug; 21(4):375- 8.

22. Meneses Falcón C. Mujer y heroína: un estudio antropológico de la heroinomanía femenina. Granada (ES): Universidad de Granada; 2001.
23. Pons Diez X. Modelos interpretativos del consumo de drogas. Polis. 2008;4(2):157-86.

24. Giancola PR. Alcohol-related aggression during the college years: theories, risk factors and policy implications. J Stud Alcohol Suppl. 2002 Mar; (14):129-39

25. García L, González MT, Egea E. Consumo de sustancias en chicas adolescentes. $2^{\mathrm{a}}$ ed. Islas Canarias (KY): Fondo Social Europeo, Instituto Canario de La Mujer; 2008. 